$\begin{gathered}\text { Revista do Departamento de Geografia } \\ \text { Universidade de São Paulo } \\ \text { www.revistas.usp.br/rdg }\end{gathered}$
Volume Especial - XVII SBGFA / I CNGF (2017)
ISSN 2236-2878

\title{
Mapeamento de Superfícies Impermeáveis em Áreas Urbanas Utilizando Imagens Índices Geradas Pelo Sistema Landsat-5 Thematic Mapper
}

\section{Mapping Impervious Surfaces in Urban Areas Using Index Images Generated From The Landsat-5 Thematic Mapper System}

\author{
Natanael Rodolfo Ribeiro Sakuno \\ Universidade Federal de Alfenas \\ natanael.sakuno@gmail.com \\ Fernando Shinji Kawakubo \\ Universidade de São Paulo \\ fskgeo@gmail.com \\ Velibor Spalevic \\ University of Montenegro \\ velibor.spalevic@gmail.com \\ Ronaldo Luiz Mincato \\ Universidade Federal de Alfenas \\ ronaldo.mincato@unifal-mg.edu.br
}

\begin{abstract}
Resumo: As áreas de superfície impermeáveis são indicativas de urbanização e por meio do mapeamento delas é possível mensurar os impactos que a urbanização gera no ecossistema urbano. $\mathrm{O}$ sensoriamento remoto constitui uma ferramenta valiosa que permite não só mapear a diversidade de uso e de cobertura vegetal como também estimar a porcentagem de cobertura impermeável. Assim, este estudo teve como objetivo estimar por meio de imagens multiespectrais do satélite Landsat-5 TM as superfícies impermeáveis existentes na mancha urbana de São José dos Campos - SP. A estimativa de superfícies impermeáveis foi feita por meio de três índices: o Normalized Difference Built-up Index (NDBI), o Normalized Difference Vegetation Index (NDVI) e o Built-up (BU). Para a validação dos resultados obtidos foram utilizadas imagens ortorretificadas do satélite Quickbird com resolução espacial de 0,5 metros.
\end{abstract}

Palavras-chave: Sensoriamento Remoto; NDBI; NDVI; Built-up.

\begin{abstract}
Impervious surface areas (ISA) are indicative of urbanization and through their mapping, it is possible to measure the urbanization impact generated on the urban ecosystem. Remote sensing is a valuable tool that enables both the mapping of land use and land cover types and estimating the percentage of impervious coverage. This study, therefore, aims at contributing to the mapping of ISA in the São José dos Campos - SP urban area, using multispectral images taken by the Thematic Mapper sensor onboard the Landsat-5 satellite. Three methods for estimating impervious surface areas were employed in this study: Normalized Difference Built-up Index (NDBI), Normalized Difference Vegetation Index (NDVI) and Built-up index (BU). The obtained results were validated using an orthorectified Quickbird image with 0.5 meter of spatial resolution.
\end{abstract}

Keywords: Remote Sensing; NDBI; NDVI; Built-up. 


\section{INTRODUÇÃO}

A relação homem natureza sempre foi um objeto de estudo que instigou pesquisadores da Geografia já que essa relação tem efeitos divergentes: de um lado o homem se beneficia com a premissa de um progresso e desenvolvimento, de outro, a natureza fica à mercê desses interesses que nem sempre são avaliados de forma a visar o bem-estar natural da Terra. Assim, é necessário compreender as pegadas ecológicas deixadas pela humanidade (SUTTON et al., 2009).

Em todo caso, há algumas décadas vem sendo suscitadas discussões sobre a vida útil que esta natureza possui sob o uso indiscriminado do homem e, por conseguinte, diversos estudos científicos vêm sendo produzidos para que se tenha uma base teórica tratando do passado dessa relação do homem com o meio natural. No entanto, além dessa discussão, é necessário evoluir as técnicas que possam permear a análise de maneira quantitativa a fim de gerar informações consistentes que possam ser debatidas na melhora qualitativa do meio ambiente.

Assim, a urbanização se insere como um evento caracterizado pela modificação antrópica extrema do meio ambiente com o intuito de torná-lo utilizável de maneira plena para as necessidades humanas. No entanto, essas modificações acabam por gerar impactos negativos para o meio ambiente, como a impermeabilização do solo, onde são substituídas as superfícies com coberturas vegetais por outras que impedem a percolação da água e o prosseguimento do ciclo hidrológico (MALLICK et al., 2013), atingindo o processo de escoamento superficial sobre a terra e também podem ser associados a problemas climáticos e no ciclo de carbono, devido ao desmatamento de grandes áreas (WENG E LU, 2008; ELVIDGE et al., 2004).

Existe uma profunda relação entre as superfícies impermeáveis e a qualidade da água de uma bacia de drenagem e seus fluxos de recepção como rios e lagos, já que o acréscimo de superfície impermeável gera um impacto na dinâmica de poluentes que passam a ser transportados pelos sistemas de saneamento básico até lençóis freáticos, afetando diretamente a qualidade de ecossistemas aquáticos e até mesmo a qualidade de vida de populações (WENG E LU, 2008). Há, com isso, impactos no processo de evapotranspiração, reduzindo os índices pluviométricos de uma determinada área urbanizada, já que grande parte da água pluvial é removida pelos sistemas de saneamento básico, diminuindo a evaporação através de calor latente, fazendo com que a radiação emitida se transforme em calor sensível, pois atinge a terra e o ar diretamente (FREITAS E DIAS, 2005).

Por conseguinte, outro fenômeno gerado é o de ilhas de calor (MALLICK et al., 2013), pois ao se modificar a cobertura do solo, tem-se uma alteração no balanço de radiação, gerando o aquecimento gradual do meio urbano que passa a refletir a radiação solar, consequentemente propagando calor, agora absorvido pela atmosfera. Além disso, o estreitamento das ruas e os altos prédios acarretam o aprisionamento da energia, aumentando a temperatura média em meio urbano (FREITAS E DIAS, 2005; ZHANG et al., 2009).

Sendo assim, em vista dos inúmeros problemas gerados pela urbanização, são necessárias abordagens do espaço urbano de forma conjunta e sistemática, tornando a aplicação do sensoriamento remoto ferramenta fundamental. Yang et al. (2010) ressaltam a importância dessa técnica para análise espacial de bacias de drenagem, afirmando que as técnicas de sensoriamento remoto possuem a inerente habilidade de prover informação temporal e espacial sobre a superfície terrestre e podem ser a única forma para monitorar em larga escala as mudanças no uso da cobertura terrestre ao longo de uma bacia de drenagem.

No entanto, uma das formas de compreender as pegadas ecológicas deixadas pela urbanização são aquelas que possam mensurar áreas de superfície impermeável (SUTTON et al., 2009) e são caracterizadas como uma superfície onde a água não consegue se infiltrar no solo e estão associadas com sistemas de transporte urbanos como ruas, rodovias, estacionamentos e calçadas e também com construções urbanas (BAUER et al., 2007).

Com isso, é concomitantemente encontrado análises tanto do espaço urbano de determinada área através da multitemporalidade espacial e da classificação de índices que podem determinar e quantificar essas áreas. Esse quadro se repete devido à crescente facilidade de uso do sensoriamento remoto, que consiste na obtenção de informações a partir da radiação eletromagnética, que é considerada uma ferramenta imprescindível na aceleração e redução de custos na obtenção de informação geográfica frente aos métodos de mapeamento tradicionais (ZHANG et al., 2009). 
A crescente geração de imagens orbitais pontuais e sistemáticas do espaço terrestre em diferentes escalas subsidiam pesquisas que, em conjunto com Sistemas de Informação Geográfica (SIG) e dados estatísticos, podem contribuir no planejamento e na aplicabilidade de políticas públicas ambientais eficazes no desordenado e crescente espaço urbano brasileiro. Assim, compreender e quantificar áreas permeáveis e impermeáveis é necessário para o planejamento das cidades consideradas futuras metrópoles brasileiras para que problemas como enchentes, ilhas de calor, impermeabilização do solo e demais impactos e danos possam ser evitados e mitigados.

\section{MATERIAIS E MÉTODOS}

O processo de expansão urbana consolidou regiões que hoje são centros metropolitanos de grande importância econômica para o país, sobretudo as localizadas no eixo Rio de Janeiro - São Paulo. No contexto paulista, a cidade de São José dos Campos (Figura 1) se destaca como uma região onde a expansão urbana atuou de forma intensiva ao longo das últimas décadas.

Segundo dados do Instituto Brasileiro de Geografia e Estatística (IBGE, 2010) o município conta com um contingente estimado de 629.921 habitantes e está localizada no interior do Estado de São Paulo, pertencente à Mesorregião do Vale do Paraíba Paulista, o município se configura como um polo técnicocientífico-industrial por conectar São Paulo e Rio de Janeiro pela Rodovia Presidente Dutra (BR - 116).

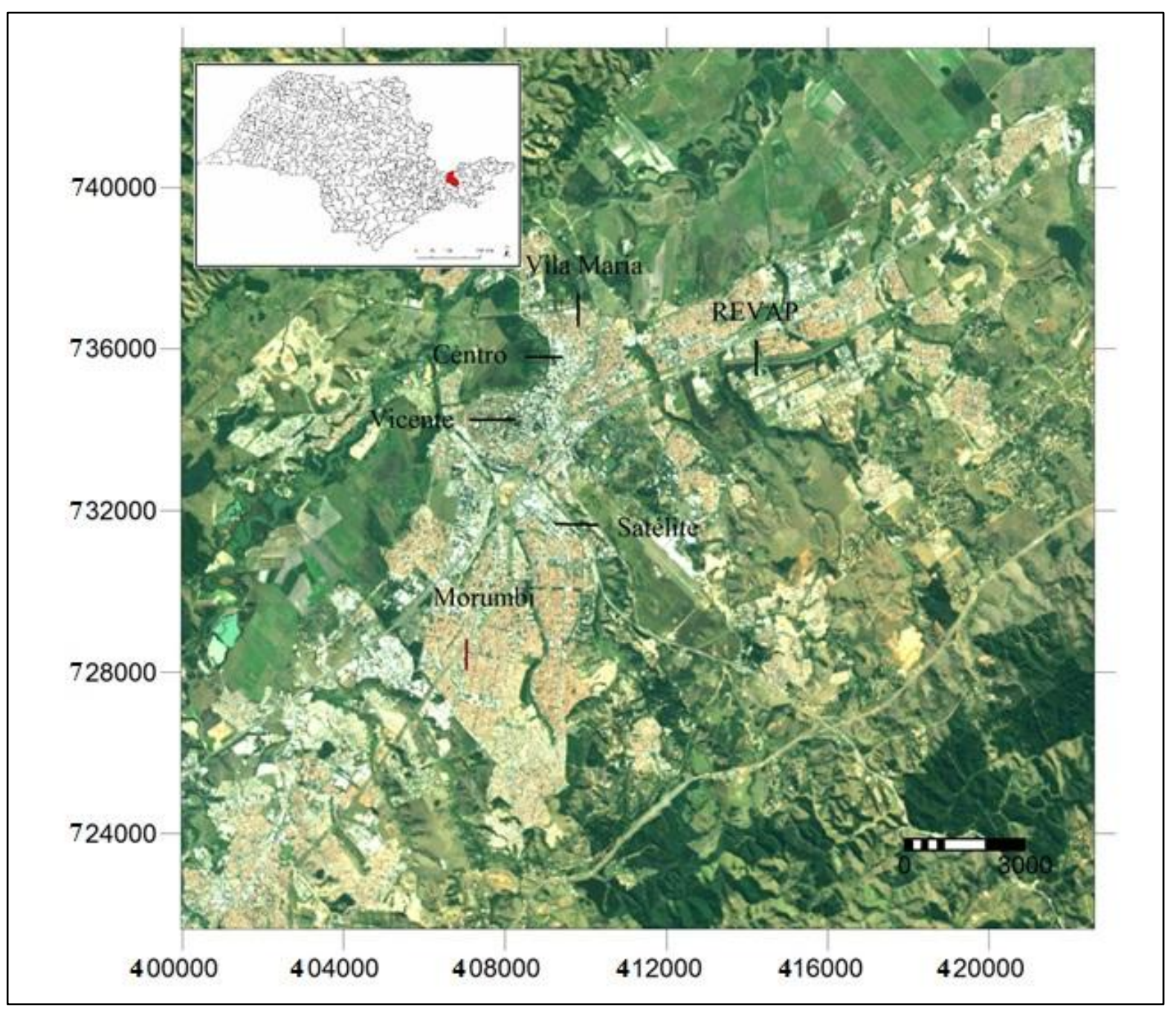

Figura 1: Localização da área de estudo e os bairros selecionados para a validação dos índices gerados. A imagem apresentada ilustra uma composição colorida real construída utilizando as bandas 3, 2 e 1 em filtros RGB. 
Inicialmente foi feito a obtenção das imagens pela plataforma do INPE, DGI (http://www.dgi.inpe.br/), depois de selecionado as imagens do Landsat 5 TM (Thematic Mapper), datado de 2010, na órbita 219 e ponto 76, foi feita a transferência das imagens em formato passível de leitura pelo software Ilwis 3.0 e, em seguida, o tratamento das imagens para obtenção dos dados.

Assim, foi gerado um sistema de coordenadas cuja função é de integrar os diferentes layers em uma mesma base de dados e, para realização e a integração dos layers, os arquivos foram georreferenciados e padronizados em um mesmo sistema de coordenadas por estudo de projeção cartográfica. Em seguida foi selecionado o retângulo envolvente, elipspóide, datum horizontal e zona.

$\mathrm{Na}$ etapa de seleção do retângulo envolvente, as coordenadas UTM utilizadas para padronização e recorte da área de estudo foram: Mín/Máx (X) 374364.5 / 434364.5 e Mín/Máx (Y) 7460859.6 / 7400859.6.

No georreferenciamento foi feita a correção geométrica por meio de pontos de controle utilizando a base cartográfica de quadras de São José dos Campos cedida pela prefeitura municipal. O erro do ajuste foi da ordem de 0,5 pixels, definidos pelo sigma a partir da identificação de pontos de controle nas bases vetoriais georreferenciadas para correção das coordenadas pelo procedimento conhecido como registro de imagens.

Após a identificação dos pontos de controle, as imagens georreferenciadas foram reamostradas pelo método do interpolador do vizinho mais próximo (KAWAKUBO et al., 2013). Esse método é utilizado porque não altera os valores de níveis de cinza e de brilho dos pixels da imagem original, importante na construção de imagens índices e na validação desses índices.

Em seguida, foram utilizados os índices que retratam informações do pixel da área construída e da vegetação, além da diferença entre os dois índices, que visa separar os índices positivos como área construída e negativos para áreas de vegetação, separando as áreas possivelmente permeáveis e impermeáveis.

O NDBI (Normalized Density Building Index) é o índice de diferença normalizada de áreas construídas (ZHA et al., 2003). Este índice permite visualizar áreas construídas nos intervalos das bandas $4 \mathrm{e}$ 5 dos canais do infravermelho próximo (NIR) e infravermelho médio (SWIR), respectivamente. Tal índice atua na diferenciação de áreas com solo exposto das áreas construídas, facilitando a classificação de padrões urbanos. Para construção do NDBI é utilizada a Equação 1.

$$
N D B I=\frac{(S W I R-N I R)}{(S W I R+N I R)} \quad(\text { Equação 1) }
$$

Em que: NDBI = Normalized Density Building Index, SWIR = reflectância na banda do infravermelho médio e NIR = reflectância no infravermelho próximo.

Já o NDVI (Normalized Density Vegetation Index) é o índice da diferença normalizada para a vegetação que permite analisar e separar os tipos de densidade de vegetação (cobertura vegetal) pela presença e ou ausência de água foliar. Informa a razão da diferença dos comprimentos de onda, onde a vegetação tem refletância alta na banda do infravermelho próximo (NIR) e no comprimento de onda de baixa reflectância da vegetação, a banda vermelha (V) (HE et al., 2010), conforme Equação 2.

$$
N D V I=\frac{(N I R-V)}{(N I R+V)} \quad(\text { Equação 2) }
$$

Em que: NDVI = Normalized Density Vegetation Index, NIR = reflectância na banda infravermelho próximo e $\mathrm{V}=$ reflectância na banda vermelha.

Para melhorar o índice NDBI, Zha et al. (2003) propuseram um índice para separação de áreas impermeáveis e permeáveis denominado de Built-up index (BU). Este é derivado de imagens contínuas do NDBI e NDVI, para que os valores positivos de NDBI indiquem as áreas construídas e os valores positivos de NDVI indiquem as áreas de vegetação, separando as áreas urbanas das áreas vegetadas e de solo exposto. A partir das imagens binárias geradas, é aplicada a Equação 3 para a extração das áreas construídas em imagem binária.

$$
B U=N D B I^{2}-N D V I^{2} \quad(\text { Equação 3) }
$$


Em que: BU = índice para separação de áreas impermeáveis e permeáveis, NDBI = Normalized Density Vegetation Index e NDVI = Normalized Density Vegetation Index .

Para validação dos resultados foram utilizadas imagens de alta resolução do Quickbird já ortorretificadas. Primeiramente, foi feito a interpretação do uso da terra nos 6 bairros pré-selecionados. A interpretação foi feita com base nos elementos de reconhecimento da fotointerpretação. Os limites de cada classe foram digitalizados e, em seguida, poligonizados utilizando um algoritmo de conversão segmento polígono. Posteriormente, as diferentes classes de uso foram reclassificadas em duas, representando as superfícies permeáveis e impermeáveis (Figura 2).

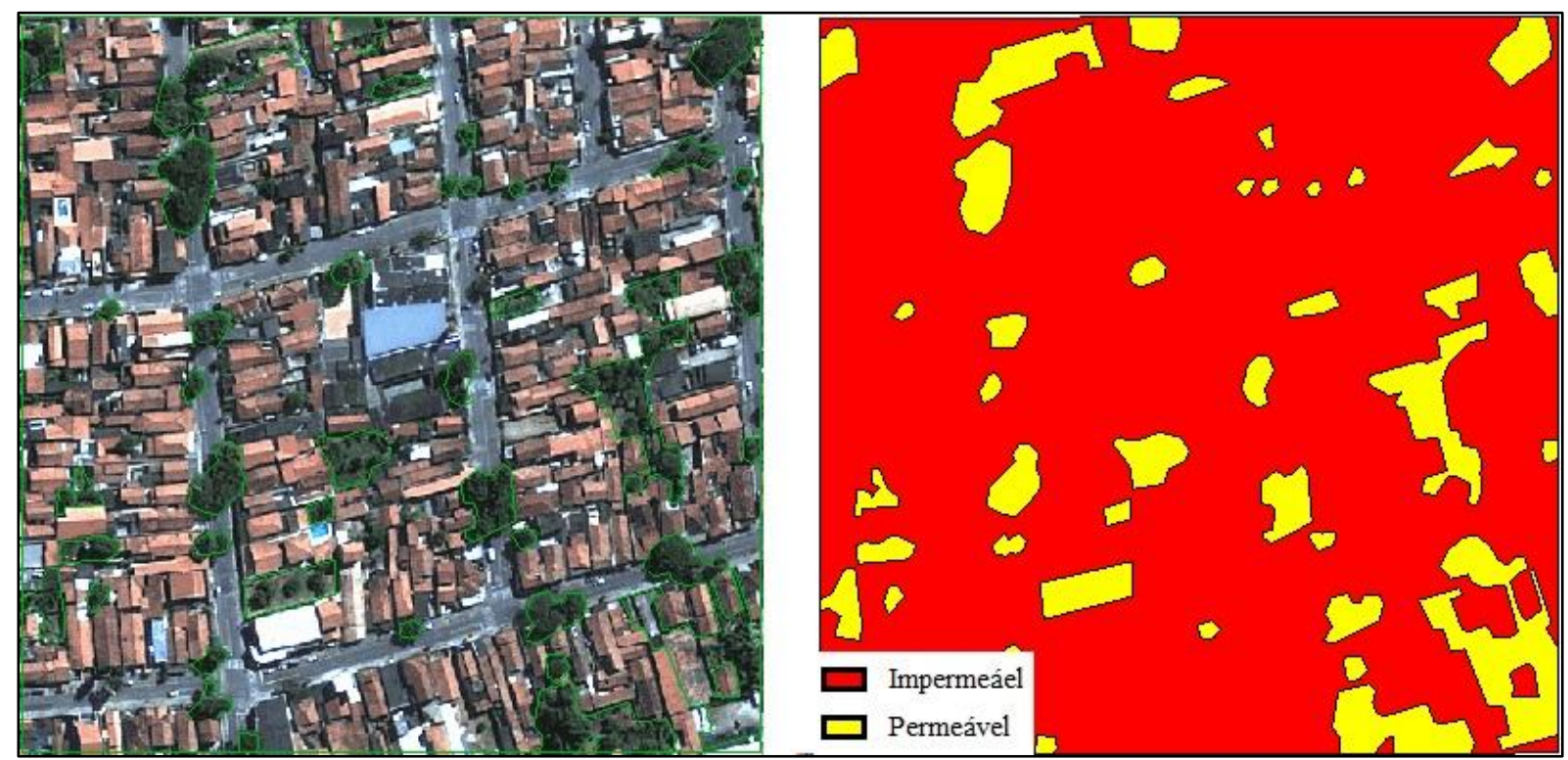

Figura 2: Composição colorida em cor real da imagem Quickbird (à esquerda) e o resultado do mapeamento contendo as classes impermeáveis e permeáveis (à direita). A área ilustrada corresponde ao bairro Vila

Maria.

Cada área teve uma imagem recortada a partir do Quickbird e os pixels divididos pela imagem referência em 100, de 1 a 100 pixels. Assim, cada pixel continha um valor que se referia à quantidade de área impermeável ou permeável que o pixel apresentava. As grades do NDBI, assim como do NDVI e do BU continham a separação de valores obtida pelos índices através das fórmulas sob a imagem do Landsat-5 TM.

A validação dos índices que representam superfícies impermeáveis foi feita utilizando a técnica de tabulação cruzada onde os pixels da imagem foram correlacionados com os valores de superfície impermeáveis obtidos por meio das imagens Quickbird. A aderência da correlação foi analisada por meio da reta de regressão e o valor de $\mathrm{R}^{2}$.

Essa reta de regressão e os valores de $\mathrm{R}^{2}$ foram obtidos pela compilação dos dados em tabelas que se correlacionavam quanto aos pixels da imagem Referência para os índices. Foi utilizado apenas a área impermeável na geração da reta, que foi ascendente em se tratando do NDBI e BU, por serem índices que retratam área construída e descendente para o NDVI, que relata a quantidade de vegetação da área.

$\mathrm{Na}$ seleção das regiões para a validação dos índices foi adotado o critério de heterogeneidade da paisagem. Ou seja, foram selecionadas áreas com diferentes padrões de uso e cobertura vegetal a fim de verificar a dinâmica dos índices e a sua variação conforme a porcentagem de áreas permeáveis e impermeáveis.

\section{RESULTADOS}

Os resultados obtidos podem ser visualizados pela comparação da tabela com as áreas analisadas (Tabela 1) e as imagens geradas (Figura 3). A variação de porcentagem para cada área ocorreu devido a diversos fatores como a diversidade de materiais presentes, a variação de superfície permeável/impermeável, 
assim como o critério de sombra adotado, em que se considerou o dossel das árvores que não se mostravam aparentes pela sombra, mas foram atribuídos e definidos como área permeável.

Tabela 1: Porcentagens das relações entre o NDBI, NDVI e BU com o método manual e a média dos 3 índices de cada área.

\begin{tabular}{|c|c|c|c|c|}
\hline Áreas & $\begin{array}{c}\text { Referência } \\
(\%)\end{array}$ & $\operatorname{NDBI}\left(\mathbf{R}^{2}\right)$ & $\operatorname{NDVI}\left(\mathbf{R}^{2}\right)$ & Bult-up $\left(\mathbf{R}^{2}\right)$ \\
\hline Centro & 81 & $65 \%$ & $77 \%$ & $76 \%$ \\
\hline Vila Maria & 86 & $16 \%$ & $33 \%$ & $36 \%$ \\
\hline REVAP & 52 & $55 \%$ & $83 \%$ & $72 \%$ \\
\hline Morumbi & 85 & $41 \%$ & $40 \%$ & $51 \%$ \\
\hline Vicente & 40 & $60 \%$ & $54 \%$ & $52 \%$ \\
\hline Satélite & 80 & $5 \%$ & $42 \%$ & $39 \%$ \\
\hline
\end{tabular}

A tabela acima apresenta o valor de área impermeável de cada uma das 6 áreas representadas na primeira coluna e também consta a variação de porcentagens que a metodologia apresentou em relação à referência manual com a obtida pelos índices. A variação de porcentagem do índice NDBI atingiu o maior valor de correlação, com $65 \%$ na área central e o menor valor de correlação no bairro satélite. Já o NDVI apresentou maior correlação na área da Refinaria Henrique Lages (REVAP), com 83\% de correlação, enquanto a menor correlação ocorreu no bairro Vila Maria da Zona Central da cidade, com 33\% de correlação. Por fim o BU também obteve o maior valor na Zona Central, com 76\%, e menor índice no bairro Vila Maria, com $36 \%$ de correlação.

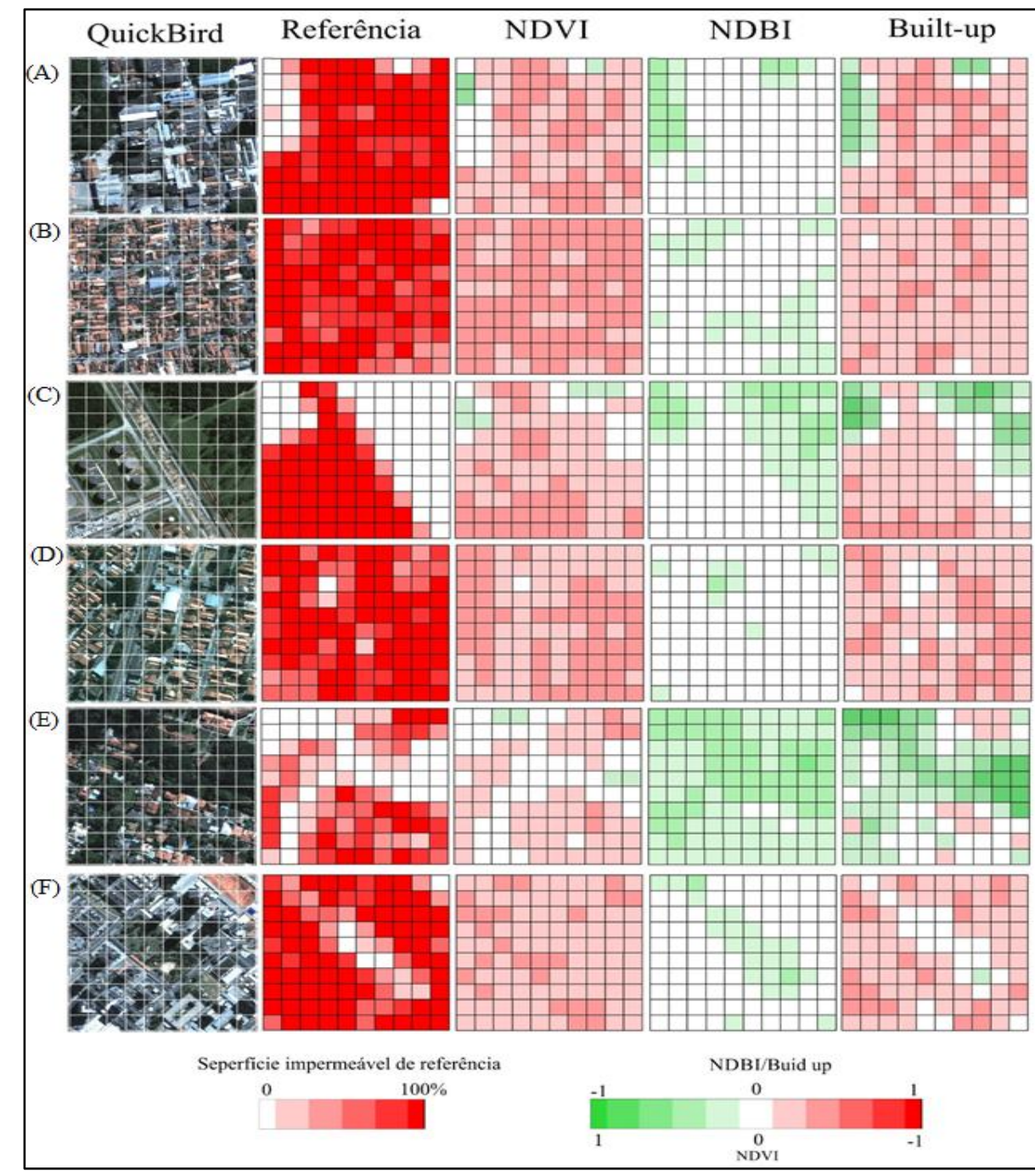

Figura 3: Comparação entre superfície impermeável calculada a partir das imagens Quickbird (1 m) e imagens Landsat-TM (30 metros) por meio dos índices NDVI, NDBI e Build-up. As áreas mapeadas 
correspondem: (A) Centro; (B) Vila Maria; (C) REVAP; (D) Morumbi; (E) Vicente e (F) Satélite. Os valores negativos podem ser interpretados como de $0 \%$ e o valor 1 como $100 \%$.

$\mathrm{Na}$ validação, que seguiu o pressuposto do método por pixel, ocorreram maiores dificuldades de compatibilizar a área urbana, por apresentar maior diversidade de materiais do que as áreas de vegetação. As construções urbanas são complexas e heterogêneas e, com isso, sofrem variação na reflexão da radiação, o que interfere na imagem obtida e pode afetar os valores dos pixels de maneira significativa (WENG E LU, 2008; FLANAGAN E CIVCO, 2001).

Quanto à exatidão da relação entre escalas, projeções e ângulos de obtenção das imagens, ocorreu erro de pixel entre os layers que resultou na readequação e reamostragem já que somente a correção geométrica não consegue atuar na escala do pixel e foi necessário a exatidão de pixel a pixel nas imagens. Problema semelhante ocorreu com Flanagan e Civco (2001), mesmo pequenos erros da relação entre a imagem registro e a do Landsat-5 TM ocasionaram em grandes variações potenciais entre o real e os valores previstos. Esse erro é menor quando se trata de áreas de bacias de drenagem ou de vegetação abundante, no entanto, em se tratando de áreas urbanas com grande percentual de área impermeável, o erro fica em evidência.

Assim, esse erro pode afetar significativamente a precisão da classificação, mesmo em uma escala relativamente grande. Segundo Flanagan e Civco (2001), a precisão pode ser afetada de forma negativa pelo erro de registro de meio pixel. As variações de precisão podem atingir até $50 \%$ do real da imagem, com $75 \%$ de desvio de precisão quando atinge metade de um pixel, ou até $100 \%$ com a mudança de um pixel completo.

A resolução necessária foi encontrada no georreferenciamento das 6 áreas especificas estudadas com dados vetoriais ortorretificados e a análise de pixel a pixel de cada área, em comparação com a obtenção manual para visualizar os possíveis erros, permitindo a reamostragem e readequação manual entre o registro e a imagem real trabalhada.

Em algumas áreas, com isso, a aplicação da metodologia foi mais eficaz, em outras, devido a diversos fatores, apresentaram uma menor compatibilização do método com os índices. Pois, em apenas um pixel de $30 \mathrm{~m}$ ou $900 \mathrm{~m}^{2}$, podem existir diferentes materiais no complexo espaço urbano já que se trata de um pixel misturado que apresenta alterações em razão da falha da escala de observação (resolução espacial) corresponder às características espaciais do alvo (WENG E LU, 2008).

Assim, a área central de São José dos Campos foi a que apresentou a maior correlação positiva. Os três índices em conjunto atingiram valores de $73 \%$ de correlação, a maior média apresentada entre as seis áreas escolhidas.

Portanto, o centro apresentou-se efetivo na proposta do método utilizado, isso se traduz pelas altas porcentagens que o $\mathrm{R}^{2}$ atingiu e que significam uma grande relação entre a poligonização de áreas impermeáveis e sua comparação com os índices que se propõe a separar áreas urbanas, de vegetação e área construída.

Os índices NDVI e BU apresentaram maior correlação de termos na área central devido às grandes proporções de área de vegetação contínua (Praça Afonso Pena). Isso demonstra que o método utilizado é válido principalmente em áreas onde há proporções contínuas de área urbana em contraposição às áreas de vegetação, já que os índices visam à resposta espectral e o método manual se baseia na observação do pesquisador e seu conhecimento prévio da área.

\section{CONCLUSÕES}

Entre os três índices, os que mais forneceram correlação positiva com o método manual foram o NDVI e o BU. Ambos apresentaram uma maior correlação em relação ao NDBI, mesmo se tratando de área urbana com maior proporção impermeável. Assim, o índice que capta a resposta espectral de vegetação foi mais sensível, pois a variação de material da vegetação é pequena em comparação a de área construída e o BU também compartilha dessa premissa já que é um índice proposto a partir do cálculo entre NDVI e o NDBI.

A variação na porcentagem em algumas das áreas foi considerada em razão da diversidade de materiais que a área urbana comporta, além do erro de estimativa ocasionado pelo deslocamento de pixels que o georreferenciamento e a reanálise não podem captar de forma plena. Mesmo que o deslocamento fosse de apenas alguns metros e não de um pixel inteiro ou de até mesmo meio pixel causou erro de validação, já 
que a área considerada impermeável ou permeável do pixel feito pelo método manual não se refere totalmente à área que o índice automático apresenta.

Outro fator preponderante na forma como os resultados se apresentaram foi a consideração do dossel e da sombra na poligonização manual, como todo o dossel é poligonizado como área permeável há uma perda de área visível que possa ser impermeável já que se trata de áreas urbanas e as árvores que se encontram em calçadas possuem uma pequena área permeável e o restante coberto por concreto ou demais materiais.

A partir da análise da variação dos resultados foi possível compreender a necessidade de se compatibilizar o método e melhorá-lo com o intuito de se obter dados satisfatórios na quantificação de superfícies impermeáveis e permeáveis e a validação dos métodos consolidados empregados. O método é eficaz em uma escala que permeie pequenas áreas e superfícies contínuas, pois quando há diversidade de materiais o método pode não ser a melhor alternativa, além da necessidade de revisar as diferenças entre os sensores e os ângulos de visada, que influenciam no posicionamento do pixel e impactam os resultados de validação.

Este estudo, a partir de técnicas de sensoriamento remoto, permitiu validar índices já consolidados, que podem subsidiar novas pesquisas e o consequente melhoramento pela escolha de imagens de outros satélites como o Landsat-8 Oli que possuem imagens já ortorretificadas.

Quantificar ou analisar as áreas impermeáveis é uma tarefa necessária, assim como validar os índices que realizam estimativas de determinadas áreas e que podem ser quanto ao grau de área construída (NDBI) e área de vegetação (NDVI), pois a técnica com o uso de dois índices demonstra a relação entre área construída e área de vegetação (BU), que permite comparar metodologias consagradas com outros métodos que podem melhorar a definição de áreas de superfície impermeável de maneira contundente.

\section{REFERÊNCIAS}

BAUER, M.E.; LOFFELHOLZ, B.C.; WILSON, B. Estimating and mapping impervious surface area by regression analysis of Landsat imagery. In: WENG, Q. (Ed.). Remote sensing of impervious surfaces. Boca Raton: CRC Press, 2007. p. 3 - 19.

ELVIDGE, C.D.; TUTTLE, B.T.; SUTTON, P.C.; DIETZ, J.B.; NEMANI, R. US constructed area approaches the size of Ohio. EOS, Transactions, American Geophysical Union, Washington, D.C., v. 85, n. 24, p. 233- 235, jun. 2004.

FLANAGAN, M.; CIVCO, D.L. Subpixel impervious surface mapping. In: PROCEEDINGS OF THE ASPRS 2001 ANNUAL CONFERENCE, apr.23-27; 2001, St. Louis, Missouri. Anais... St. Louis, Missouri: American Society for Photogrammetry and Remote Sensing, 2001.

FREITAS, E.D.; SILVA, P.L.D. Alguns efeitos de áreas urbanas na geração de uma ilha de calor. Revista Brasileira de Meteorologia, São José dos Campos, v. 20, n.3, p.355-366, 2005.

HE, C.; SHI, P.; XIE, D.; ZHAO, Y. Improving the normalized difference built-up index to map urban builtup areas using a semiautomatic segmentation approach. Remote Sensing Letters, Novi Sad, v. 1, n. 4, p. 213-221, mar. 2010.

IBGE. Instituto Brasileiro de Geografia e Estatística. Censo Populacional 2010. Acesso em 12 de fevereiro de 2015, disponível em <http://www.ibge.gov.br /home /estatistica/ populacao/censo2010/>

KAWAKUBO, F.S., MORATO, R.G., LUCHIARI, A. Use of fraction imagery, segmentation and masking techniques to classify land-use and land-cover types in the Brazilian Amazon. International Journal of Remote Sensing, Bristol, v. 34, n. 15, p. 5452-5467, mar. 2013.

MALLICK, J.; RAHMAN, A.; SINGH, C.K. Modeling urban heat islands in heterogeneous land surface and its correlation with impervious surface area by using night-time ASTER satellite data in highly urbanizing city, Delhi-India. Advances in Space Research, Delhi, v. 52, n. 4, p. 639-655, aug. 2013.

SUTTON, P.C.; ANDERSON, J.S.; ELVIDGE, C.D.; TUTTLE, B.T. Paving the planet: impervious surface as proxy measure of the human ecological footprint. Progress in Physical Geography, Thousand Oaks CA, v. 33, n. 4, p. 510-527, aug. 2009. 
WENG, Q.; LU, D. A sub-pixel analysis of urbanization effect on land surface temperature and its interplay with impervious surface and vegetation coverage in Indianapolis, United States. International Journal of Applied Earth Observation and Geoinformation, Novi Sad, v. 10, n. 1, p. 68-83, feb. 2008.

YANG, F.; MATSUSHITA, B.; FUKUSHIMA, T. A pre-screened and normalized multiple endmember spectral mixture analysis for mapping impervious surface area in Lake Kasumigaura Basin, Japan. ISPRS Journal of Photogrammetry and Remote Sensing, Tsukuba, v. 65, n. 5, p. 479-490, sept. 2010.

ZHA, Y.; GAO, J.; NI, S. Use of normalized difference built-up index in automatically mapping urban areas from TM imagery. International Journal of Remote Sensing, London, v. 24, n. 3, p. 583-594, 2003.

ZHANG, X.; ZHONG, T.; WANG, K.; CHENG, Z. Scaling of impervious surface area and vegetation as indicators to urban land surface temperature using satellite data. International Journal of Remote Sensing, Bristol, v. 30, n. 4, p 841-859, feb. 2009. 